\title{
Profit Profiles in Correlated Markets
}

\author{
Ingve Simonsent and Kim Sneppen \\ Nordic Intitute for Theoretical Physics - NORDITA \\ Blegdamsvej 17, DK-2100 Copenhagen Ø \\ DENMARK \\ (October 28, 2018)
}

\begin{abstract}
We consider a financial market where the asset price follows a fractional Brownian motion. We introduce a family of investment strategies, and quantify profit possibilities for both persistent and antipersistant markets.
\end{abstract}

Financial time series have been studied for a long time. What type of random process they resemble is still debated, and different suggestions have been made; In 1900 the French mathematician L. Bachelier (Bachelier 1900) suggested that asset prices might be described by what today is known as a random walk (Feder 1988). Such a walk is a random process where the increments are uncorrelated. However, today we know this is not the whole story. There are examples of financial time-series that exhibits correlations (Mantegna \& Stanley 2000, Bouchaud \& Potter 2000, Peters 1994, Peters 1996, Mulligan 1997). In particular both correlations (Peters 1996, Mulligan 1997) and recently also anti-correlations (Mulligan 1997) have been reported for various types of financial time series.

Correlated markets are at odds with an efficient market, as it may allow for arbitrage opportunities. This should be the case both for short term correlations, as well as long term scale invariant fluctuations (where transaction costs can be neglected). This paper address the amount of arbitrage as function of correlations, and thus quantify the degree of non-equilibrium and possible profits, for different employed strategies, a given long time correlation corresponds to. For a record of real markets with significant correlations, please consult (Mulligan 1997, Neely 1997, Holst \& Zebrowska 2000) (and references therein)

The price model that we are going to consider is a fractional Brownian motion, i.e. a self-affine process (Feder 1988). Such a process is characterized by an exponent, $H$, termed the Hurst exponent (Hurst 1951), where typically $0<H<1$.

Let $p(t)$ denotes the price of an asset at time $t$. The asset could be a stock, a currency or some commodity. That $p(t)$ is self-affine means that fluctuations on different timescales can be rescaled, so that the behaviour of $p(t)$ is statistically equivalent to the behaviour of $\lambda^{-H} p(\lambda t)$ where $\lambda$ is any positive number. For $H=1 / 2$ we have the uncorrelated random walk of Bachelier.

Since it will be useful later, let $t=0$ be the present time, and let us consider the correlation function between future and past price increments $\Delta p(t)=p(t)-p(0)$ and $\Delta p(-t)$ :

$$
C_{H}(t)=\frac{\langle-\Delta p(-t) \Delta p(t)\rangle}{\left\langle[\Delta p(t)]^{2}\right\rangle},
$$

where $\langle.\rangle=.\frac{1}{N} \sum_{1}^{N}$.. denotes the (arithmetic) average over all considered investment situations for fixed $H$ (and investment strategy), and thus $\left\langle\Delta p(t)^{2}\right\rangle=\left\langle\Delta p(-t)^{2}\right\rangle$. A remarkable feature of a fractional Brownian motion is that the correlation function, Eq. (1a), is time-independent and only depends on the Hurst exponent $H$ (Feder 1988):

$$
C_{H}(t)=2^{2 H-1}-1 .
$$

Thus an ordinary Brownian motion with $H=1 / 2$ have $C_{H=1 / 2}(t)=0$ whereas any larger (smaller) $H$ implies correlation (anti-correlation). I.e. if $H>1 / 2$ then $C_{H}>0$ and thus the sign of the past price difference $-\Delta p(-t)=$ $p(0)-p(-t)$ is most likely to be maintained for $\Delta p(t)$. Therefore for $H>1 / 2$ the stochastic process is most likely to keep the trend of the past. Similarly if $H<1 / 2$ the sign of the price difference is most likely to change at each subsequent time interval. Furthermore, the propability of change is independent of the length of the considered time

${ }^{*}$ Fax: $+45+4535389157$ 
interval. Hence, a process with $H>1 / 2$ is denoted a persistent fractional Brownian motion, while a process with $H<1 / 2$ is referred to as anti-persistent. In the financial literature the latter case $(H<1 / 2)$ is often referred to as a mean-reverting process.

To study profit opportunities in a fractional Brownian market, we need to define a strategy for when to (and not to) make an investment. In accordance with the notion of persistence and anti-persistence, we use a strategy that is based on the price history: Assume that we have an investment horizon $T$, meaning that after purchasing an asset at time $t=0$ the investor sticks to his investment until time $t=T$ at which point the asset is sold. We will evaluate strategies, assuming that there is only one option to buy or not to buy at the beginning of each interval. If we do not buy, nothing happens. If, however, one buys, one is bound to sell at the end of the interval. Buying or not buying at the next interval, is considered a separate independent event.

The choice of strategy, $\Pi_{\nu}$, is motivated by Eqs. (1). For an investment horizon $T$ we make a choice at $t=0$ based on the asset price at $t=-T$ : If the market is persistent $(H>1 / 2)$, one invests at $t=0$ only if the price went up from $t=-T$ to $t=0$, i.e. if $-\Delta p(-T)=p(0)-p(-T)>0$. Similarly if the market is anti-persistent $(H<1 / 2)$ one invests at time $t=0$ only if $-\Delta p(-T)<0$, i.e. if the price over the chosen horizon $T$ (in the past) has decreased. The above strategy (obtained by setting $\nu=0$ in the formulae below) can be expressed in terms of the amount invested at time $t=0$ :

$$
\Pi_{\nu}(\Delta p(-T))=\theta(-(H-1 / 2) \Delta p(-T)) \cdot \frac{|\Delta p(-T)|^{\nu}}{\left\langle|\Delta p(-T)|^{\nu}\right\rangle}
$$

where $\theta(x)=1$ for $x \geq 0$, and $\theta(x)=0$ for $x<0$. We will later return to other choices for the parameter $\nu$. In Eq. (2) the $\theta$-function signifies to invest $(\theta=1)$, or not-invest $(\theta=0)$, while the last term define the investment size for a particular past. If we always invest the same amount independent of the past, we have $\nu=0$ so that the last term of Eq. (2) is constant (and equal to one). If $\nu>0$ the size of the investment depends on the past change of $p(t)$. For example if $\nu=1$ the amount we invest is proportional to $\Delta p(-T)$ whereas higher $\nu$ values focus transactions on fewer big investments.

To measure the utility of a strategy $\Pi_{\nu}(\Delta p(-T))$ we note that the profit for an investment (occuring only when $\theta=1$ in Eq. (2)) is

$$
W_{\nu}(T)=\frac{|\Delta p(-T)|^{\nu}}{\left\langle|\Delta p(-T)|^{\nu}\right\rangle} \cdot \Delta p(T) .
$$

If the price fluctuations are increased, the potential profit (or loss) is bigger. In our fractional Brownian market the fluctuations increase with the duration of the investment horizon. Therefore the profit histograms are expected to broaden when $T$ is increased (see inset in Fig. 2). In order to compare the performance of the various strategies at different horizons we examine profits in units of the typically price fluctuations at the given investment horizon: $\sigma(-T)=\sqrt{\left\langle(p(-T)-p(0))^{2}\right\rangle} \propto T^{H}$ for a stochastic process with Hurst exponent $H$. Hence it is natural to consider the probability distribution of $W_{\nu}(T) / \sigma(-T)$.

To obtain the distribution of profit we need to generate a market. Fractional Brownian price processes were generated by the method described in Ref. (Feder 1988) which ensures Gaussian increments of the process. For $H=0.4$ an example is shown in Fig. 1. We have also considered versions of the generator described in Ref. (Feder 1988) which gives raise to non-Gaussian increments, e.g. power-laws ( n.d.a), and different generators (Prakash, Havlin, Schwartz \& Stanley 1992, Simonsen \& Hansen n.d.), but these different choices seem not to affect the general conclusions drawn in this paper. The length of the used time series was $T_{\max }=2^{14}=16384$, equally divided between past and future. To avoid influence from periodic boundaries we only consider horizons $T$ well below $T_{\text {max }} / 2$.

In the inset of Fig. 2 we show, for an anti-persistent market characterized by Hurst exponent $H=0.3$ (and strategy $\nu=0$ ), the profit probability distributions for the investment horizons $T=8,32,128,512$. The corresponding distributions for the renormalized profits, $W_{0}(T) / \sigma(-T)$, still with $H=0.3$, are shown in the main part of Fig. 22. They demonstrate data collapse for the different investment horizons. This teaches us that any time-scale provides the same profit profile, if we measure this in units of the typical price fluctuations on that time-scale (and can neglect transaction costs). This fact simplifies our discussion, as we can deal with profit in terms of only fractions of the typical price fluctuations. The vertical dashed lines in Fig. 2 separate the profit-region (to the right) from the lossregion (to the left). The asymmetry testifies to a possible mean profit, which we for Hurst exponent $H=0.3$ find to be $\left\langle W_{0}(T) / \sigma(-T)\right\rangle_{P}=0.35$ where $\langle\cdot\rangle_{P}$ is used to indicate the mean relative to the distribution $P$ (see Ref. ( n.d. $\left.b\right)$ ). We find similar data-collapses for other Hurst exponents between 0 and 1. Furthermore, in Fig. 3 the collapsed curves, for different $H$, show that the profit distribution exhibits fatter tails for larger Hurst exponents. We also observe that only $H=1 / 2$ gives raise to a symmetric distribution, whereas all other markets allow for profit.

The above analysis was done for a $\nu=0$ strategy, i.e. many equally sized investments. If one instead choose to focus the investments, that means to use a $\nu>0$ strategy, the profit would be different (see Fig. 4). However, 
the distributions ( n.d.b) of $W_{\nu}(T) / \sigma(-T)$ (results not shown), for different $\nu$ and given $H$, are rather similar. The main difference between the different strategies $\nu$ comes about due to an increased asymmetries (for $H \neq 1 / 2$ ). This asymmetry is quantified by the average profit $\left\langle W_{0}(T) / \sigma(-T)\right\rangle_{P}$. In Fig. 国 this average profit is summarized for a number of markets and a number of different strategies. For any $\nu$ we observe profit possibilities for both $H<1 / 2$ and $H>1 / 2$. For small deviations from a perfect Brownian market the profit increases linearly with the distance to $H=1 / 2$. By examining different $\nu$ we further observe that larger $\nu$ provides us with larger average profit. Thus, if the average is our only concern we should invest only when there has been extreme price changes in recent past. However, the cost of higher $\nu$ is a higher probability for big losses (and gains) simply because the invested capital increases with $\nu$. This fact was observed (result not shown) as fatter and fatter tails for the total wealth distribution. Notice, however, that the probability for big losses per invested dollar is more or less independent of $\nu$.

Finally we consider the role of transaction costs. Until now this has been neglected, as it could be for a long time investment. However, since the profit defined above always scales with the spread in prices, $W(T) \propto \sigma(T) \propto T^{H}$, the profit per time unit $W(T) / T \propto T^{H-1}$ favors (if we neglect transaction costs) very short investments (since $H<1$ ). The only limit to short term investments is transaction costs, that is a fixed fraction $r$ of every investment. With transaction costs the net profit for an investment $W_{\nu}(r, T) \propto(\Delta p(T)-r)$ where $\Delta p(T)$ here is defined as the relative price change in order to be on the same scale as the transaction cost $r$. In principle there is of course transaction costs at both beginning and end of each trade, but that would not change the functional form (i.e. $\Delta p-r \rightarrow(1-r) \Delta p-2 r)$. With transaction costs the average $W_{\nu}(r, T)$ is negative at small $T$ and $\left\langle W_{\nu}(T)\right\rangle / T$ has a maximum at some finite time $T$ that depends on Hurst exponent, as well as $\nu$ and $r$. This maximum sets the optimal investment horizon in a correlated market.

In summary, we have considered the possibility of making profit in a fractional Brownian market. It is noted that one can make profit for all cases where $H \neq 0.5$, and it is found that the average profit increases with the willingness to bet on extreme variations.

We would like to thank P. Bak for initially drawing our attention to the problems addressed in this letter.

(n.d.a). In particular we considered $1 / x^{4}$ and Lorentzian (Cauchy) distributed increments, where the latter distribution asymptotically goes like $1 / x^{2}$. The former distribution was recently observed in economic data by P. Gopikrishnan et al., Phys. Rev. E. 62, R4493 (2000).

(n.d.b). According to its definition, the invested amount varies from transaction to transaction when $\nu>0$. Hence the profit we make by applying different stratigies can not directly be compared. In order to address this, we will for $\nu>0$ by the profit distribution $P(x)$ understand the distribution of profit per unit (invested) capital. By doing so the performance of the different strategies can readily be compared.

Bachelier, L. (1900), Thèorie de la Speculation, PhD thesis, Sorbonne, Paris.

Bouchaud, J.-P. \& Potter, M. (2000), Theory of Financial Risk: From Statistical Phsyics to Risk Management, Camebridge University Press, Cambridge.

Feder, J. (1988), Fractals, Plenum, New York.

Holst, J. \& Zebrowska, M. (2000), 'Recurrence plots and hurst exponents for financial markets and foreign-exchange data', International Journal of Theoretical and Applied Finance 3, 419.

Hurst, H. (1951), 'Long-term storage capacity of reservoirs', Transactions of the American Society of Civil Engineers 116, 770. Mantegna, R. N. \& Stanley, H. E. (2000), An Introduction to Econophysics; Correlations and Complexity in Finance, Camebridge University Press, Cambridge.

Mulligan, R. F. (1997), 'A fractal analysis of foreign exchange markets', Advances in Economic Research 6, 33. (http://www3.wcu.edu/ $\sim$ mulligan/fractal.html).

Neely, C. (1997), 'Is technical analysis in the foreign exchange market profitable? a generic programming approach', Journal of Financial and Quantative Analysis 32, 405.

Peters, E. E. (1994), Fractal Market Analysis, Wiley \& Sons, New York.

Peters, E. E. (1996), Chaos and order in the Capital Markets: a New View of Cycles, Prices and Market Volatility, Wiley \& Sons, New York.

Prakash, S., Havlin, S., Schwartz, M. \& Stanley, H. (1992), 'Structural and dynamical properties of long-range correlated percolation', Phys. Rev. A 46, R1724.

Simonsen, I. \& Hansen, A. (n.d.), A fast algorithm for generating long self-affine profiles, Submitted Phys. Rev. E. 


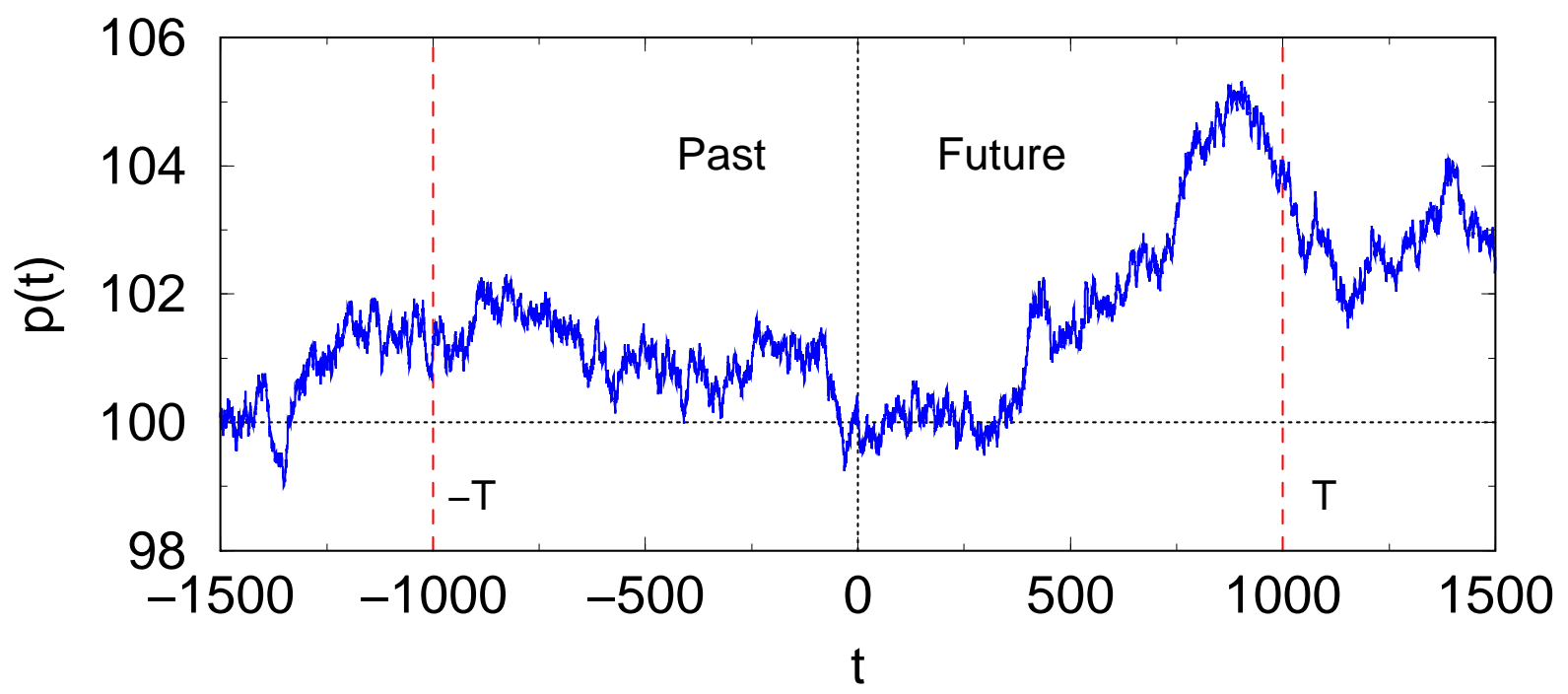

FIG. 1. An example of a price time series with Hurst exponent $H=0.4$. With the strategy described in the text, one would for time horizon $T=1000$, buy at $t=0$ because the price went down in the past and thus is more likely to increase in the future.

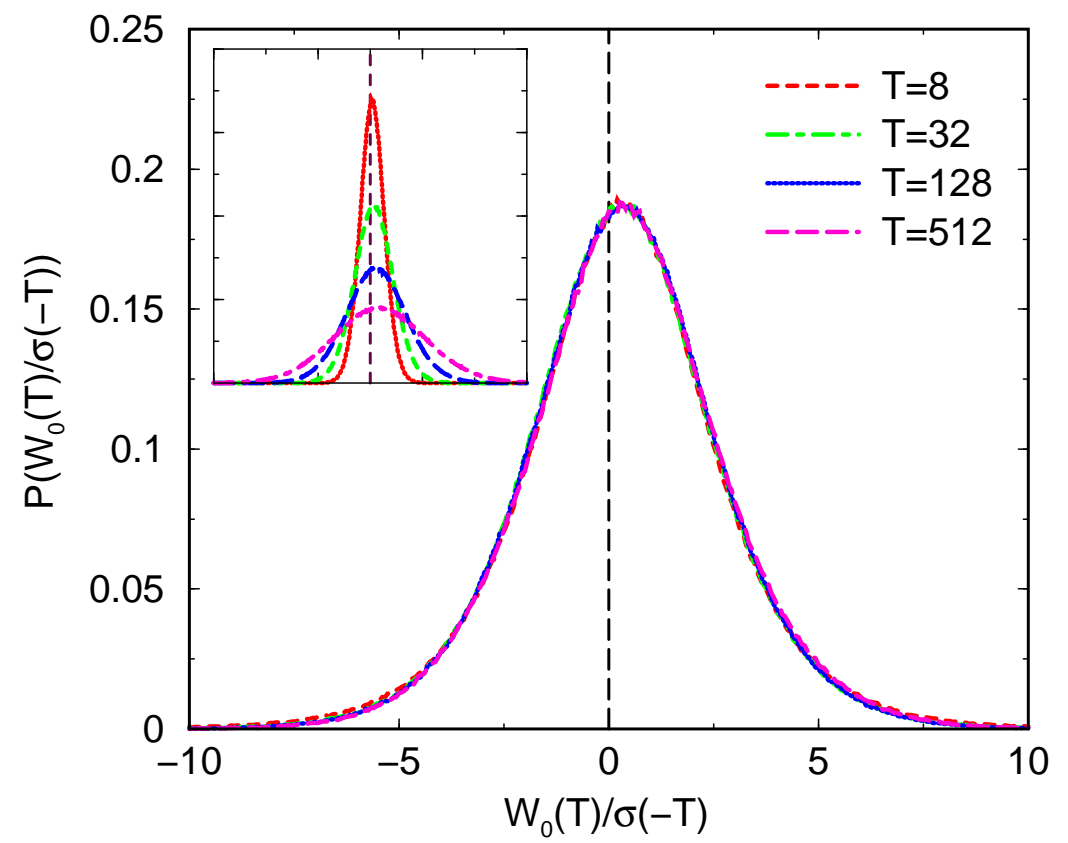

FIG. 2. The profit distribution for an anti-persistent market $(H=0.3)$ with different investment horizons $T$ (and fix strategy $\nu=0)$. The figure shows the distribution of $W_{0}(T) / \sigma(-T)$ while the inset shows the distribution of $W_{0}(T)$. 


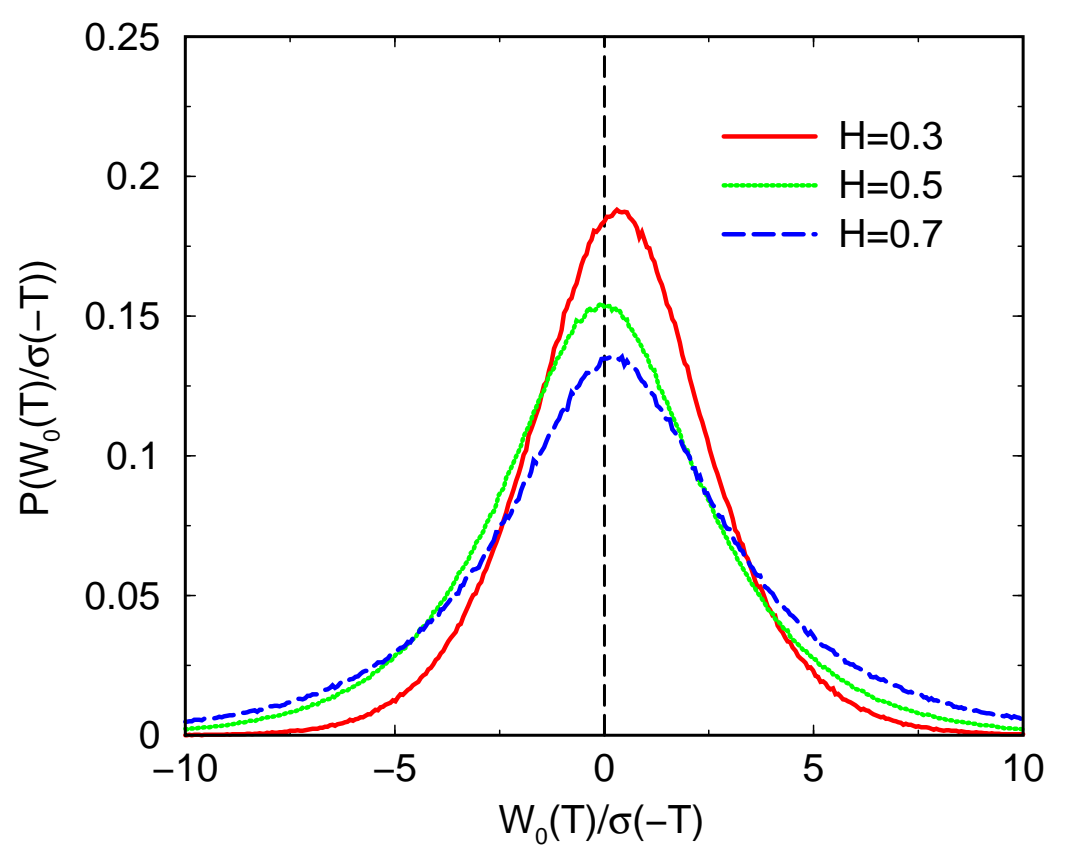

FIG. 3. The distributions of $W_{0}(T) / \sigma(-T)$ for markets with different Hurst exponents $H$ (and investment strategy $\nu=0$ ).

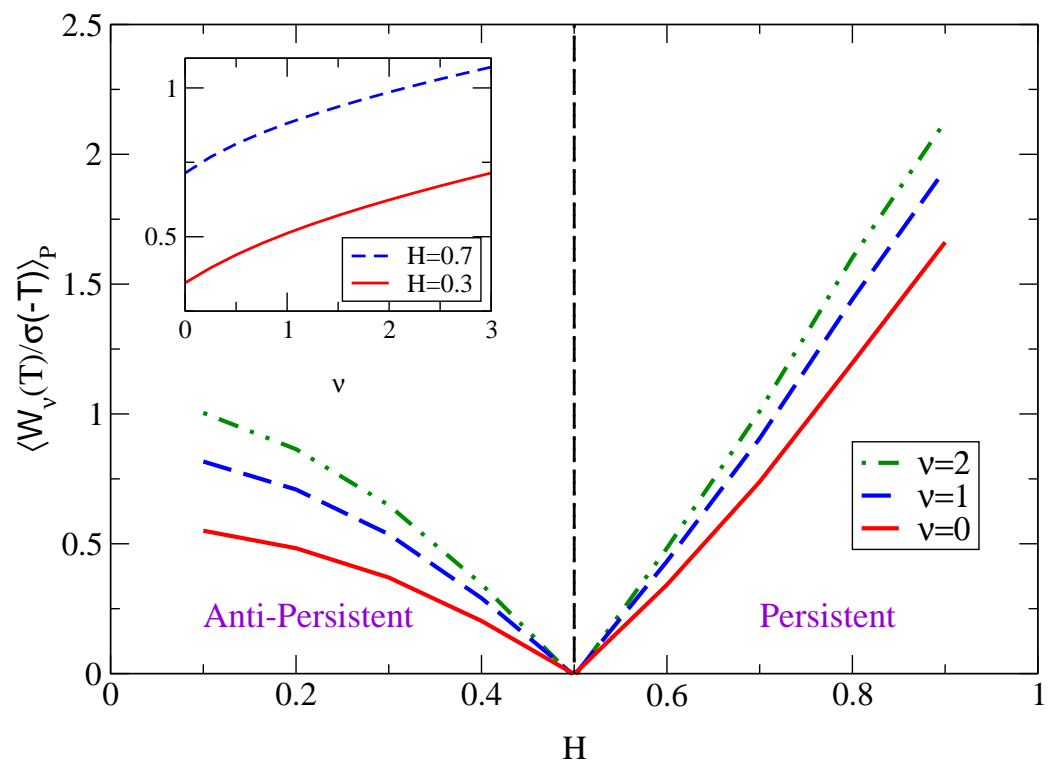

FIG. 4. The average profit $\left\langle W_{\nu}(T) / \sigma(-T)\right\rangle_{P}$ vs. Hurst exponent, $H$, for three different choices of strategy. The inset shows the same quantity for $H=0.3$ and 0.7 , but now as a function of the invetsment strategy $\nu$. 